\title{
Autism spectrum disorder classification on electroencephalogram signal using deep learning algorithm
}

\author{
N. A Ali ${ }^{1}$, A.R Syafeeza ${ }^{2}$, A. S Jaafar ${ }^{3}$, M.K Mohd Fitri Alif ${ }^{4}$ \\ 1,2,3Faculty of Electronics and Computer Engineering, Universiti Teknikal Malaysia Melaka, Hang Tuah Jaya, 76100 \\ Durian Tunggal, Melaka, Malaysia \\ ${ }^{4}$ Control and Mechatronics Engineering department, Faculty of Electrical Engineering, Universiti Teknologi Malaysia, \\ 81300, Johor Bahru, Johor, Malaysia
}

\begin{tabular}{l}
\hline \hline Article Info \\
\hline Article history: \\
Received Nov 10, 2019 \\
Revised Feb 6, 2020 \\
Accepted Feb 17, 2020
\end{tabular}

Keywords:

Autism spectrum disorder Deep learning

Electroencephalogram

\begin{abstract}
Autism Spectrum Disorder (ASD) is a neurodevelopmental that impact the social interaction and communication skills. Diagnosis of ASD is one of the difficult problems facing researchers. This research work aimed to reveal the different pattern between autistic and normal children via electroencephalogram (EEG) by using the deep learning algorithm. The brain signal database used pattern recognition where the extracted features will undergo the multilayer perceptron network for the classification process. The promising method to perform the classification is through a deep learning algorithm, which is currently a well-known and superior method in the pattern recognition field. The performance measure for the classification would be the accuracy. The higher percentage means the more effectiveness for the ASD diagnosis. This can be seen as the ground work for applying a new algorithm for further development diagnosis of autism to see how the treatment is working as well in future.
\end{abstract}

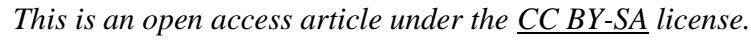

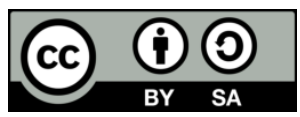

\section{Corresponding Author:}

Nur Alisa Ali,

Department of Computer Engineering,

Faculty of Electronics and Computer Engineering, UTeM,

76100 Hang Tuah Jaya, Malacca, Malaysia.

Email: alisa@utem.edu.my

\section{INTRODUCTION}

Autism Spectrum Disorder (ASD) is a syndrome that adversely affect a child where the behavioral symptoms start to appear during the first year of life [1]. This early childhood onset includes symptoms such as lack in social interaction and very slow language skills development as stated by researchers [2-3]. A continuous characters and behavioral assessment is conducted by specialist in order to detect autistic presence in a child. A documented analysis done by pediatrics stated that, an autistic child at approximately 24 months, are still unable to produce two meaningful word that do not involve imitating and repeating. Despite so much research being conducted, the exact factors to why this disorder occurs remain unanswered. As of why this atypical behavior is very difficult to detect is maybe due to the barely noticeable changes of the primary neural impairment itself.

The relation of ASD with EEG signal is there is a significant decline of EEG complexity perceived in autistic child. The noteworthy differences were observed between brain region in the right hemisphere and the central cortex represented by [4]. EEG signals are electrical voltage triggered on the electrodes by brain electromagnetic signals (BEMS) [5]. At the hospital, a specialized technician's measures, marks and put 
about 16-25 electrodes on the patients. Many research shows that the EEG signals, which consists of Alpha, Beta, Gamma, Delta and Theta, of ASD child is not the same as compared to non-ASD child [6].

Deep Learning is a subset of machine learning. As mentioned by researchers [7-8], deep learning includes the use of a lot of hidden neurons and layers, usually more than two, as an architectural benefit, in addition with new training platform; when data availability increases, deep learning systems can develop gradually and fill in the gaps where human interpretation is not possible. Regardless it being machine or deep learning, both consists of supervised learning and unsupervised learning. Supervised learning is developing predictive method based on input and output data. While unsupervised learning is grouping and interpreting data based on input data only. However, the study conducted by [9] mentioned that deep learning can be an excellent tool to be applied in clinical brain imaging. This is further supported by studies conducted by [10] stating that deep learning is a very reliable method to quickly address patient health condition by classifying of diseases, delete redundancy but at the same time maintains correctness and precision of disease identification. Eventually, the process of detecting becomes diseases less time consuming. Health care personnel can be certain on their diagnosis in the decision-making stage.

This paper is organized as follows: The next section describes the proposed approach and the database used. The results and analysis are shown in Section 3. Finally, Section 4 concludes this paper.

The number of children detected with Autism Spectrum Disorder (ASD) has been increasing in the past few years as stated by the Ministry of Health, Malaysia. Attention has been brought to this neurodevelopmental impairment disease due to the unknown cause of this disorder. Diagnosing ASD before the age of three is very challenging. This is because; ASD is highly associated with either the over-abundance or very low neuron connection of the brain wires. However, the formation of either the over-abundance or very low neuron connection during child growth is a very slow progress that it is hardly noticeable [11]. The situation is much more alarming since there is not yet a quantifiable technology used to exactly address this disorder.

ASD is not exactly genetically related, thus specialist cannot exactly predict if the child is autistic or not since birth. Many real-life cases show that if a child has autistic siblings that will not necessarily mean the child will be autistic as well. Furthermore, according to Mythili [12], each child with autism shows very distinguishable behavioral symptoms. For example, some child is very well conversed, while some barely utters words. Some child with autism is very much attached and some just did not know how to express emotions. Some autistic child even has the same behavior as normal child with very minimal behavior difference. There is a very thin barrier that separates the normal with autistic. Thus, these small barriers of behavior differences are the main challenges that keep researchers' attention to this syndrome.

Electroencephalogram (EEG) is a method used in purpose to find out the brain function regardless the mental states of the individuals [13]. EEG provides robust parameters where it can examine the brain activity at rest state and active state. EEG can also show which part of the brain is active when doing specific tasks and the effect of those specific tasks. There are five types of frequency bands associated with EEG signal namely the alpha, beta, gamma, theta and delta. As aforementioned each of this brain wave has their own frequency range and each is associated with its own physiological characteristics which governs its own cognitive properties. During EEG recording, patient will be shown series of simulation while sitting down. All these simulations have a certain way of affecting all the five frequency bands to stimulate. In child with ASD, all these five types of frequency band are interrupted and does not show result in accordance with the given simulation.

The Alpha band is known to be available when a person is in relaxed mood and are awake. They are also associated with timing and cognitive inhibition. Next, the beta bands are engaged with alertness, active task involvement and motor behavior. The gamma band is an early sensory respond, where it helps with feature binding in sensory processing. Finally, the delta band is active during deep sleep and event that is related to slow waves such as detection of attention and wakefulness. The table computes the EEG signal waveform of human being is shown in Table 1. While Figure 1 illustrates sample of EEG signal from a normal person.

Table 1. EEG Brain Signal of Normal Human Being [14]

\begin{tabular}{ccc}
\hline Level & Frequency range & Approximate EEG label \\
\hline 1 & $64-128 \mathrm{~Hz}$ & High Gamma \\
2 & $32-64 \mathrm{~Hz}$ & Gamma \\
3 & $16-32 \mathrm{~Hz}$ & Beta \\
4 & $8-16 \mathrm{~Hz}$ & Alpha \\
5 & $4-8 \mathrm{~Hz}$ & Theta \\
6 & $0-4 \mathrm{~Hz}$ & Delta \\
\hline
\end{tabular}

Int J Artif Intell, Vol. 9, No. 1, March 2020: 91 - 99 
Across many studies, it is shown that a child with ASD is consistent in producing atypical neural activity during the frequency band simulation. Hence, EEG signal reveals high hope in seeking more findings to localize the exact area inside the brain where the alteration begins. This is further supported by [14], stating that by calculating how quick the brain react to visual and audio stimuli will positively impact in classifying autism and diagnose the disorder earlier.

Deep learning combines feature extraction and classification process [15]. Feature extraction is the process of producing a dense but highly meaningful digital presentation of someone fundamental biometric attribute. In typical machine learning, the important feature needs to be manually extracted and classify using one or more classifier algorithm. In order to extract features, there are two most popular methods used which are the filter methods and the wrapper methods. An example of filter methods and wrapper methods are chi squared test and recursive feature elimination algorithm respectively. As for classifier, most common classifiers are Fisher Linear Discriminant Analysis (FLDA), Random Forest and Support Vector Machine. In many research, in order to obtain the best classification accuracy, two or more classification algorithm will be used [16]. Thus, it is agreed that since deep learning can successfully combine feature extraction and classifier together, it can decrease the experimenting and selecting features process.

One of the most popular deep learning methods is the Convolutional Neural Network, which is also called as CNN [17]. As mentioned before, like any other deep learning method, the feature that is needed to be extracted does not have to be outlined. The CNN algorithm will automatically extricate the most distinguishing characteristics without including the optimization and propagation steps. This is made possible even without using feature extractor due to the training of data step present in the CNN algorithm. The examples of applications utilized CNN through studies by [18-21].

Deep learning particularly $\mathrm{CNN}$ is deployed due to the capability of the algorithm to actually recognize unique features of electrical brainwave pattern from EEG signal. Many studies have shown success in integrating EEG signal into the CNN algorithm. EEG is non-linear and highly intricate signal that records important data. This data portrays the differences of one human being to another. Since EEG is very complex and non-linear in nature, many linear classifier methods could not accurately detect this signal as EEG. Thus, $\mathrm{CNN}$ has become increasingly popular and is becoming a highly preferred new technique in signal detection and classification. The theory of CNN is mainly inspired by the brain of human being. CNN is a neural network that consists of multilayer perceptron (MLP). Each multilayer perceptron serves its own special purposes with its own arrangement of execution as shown in Figure 2. In all MLP, there must be an input layer which comes from an input data, a minimum of one hidden layer and finally an output layer that will predicts the output from the input layer [22].
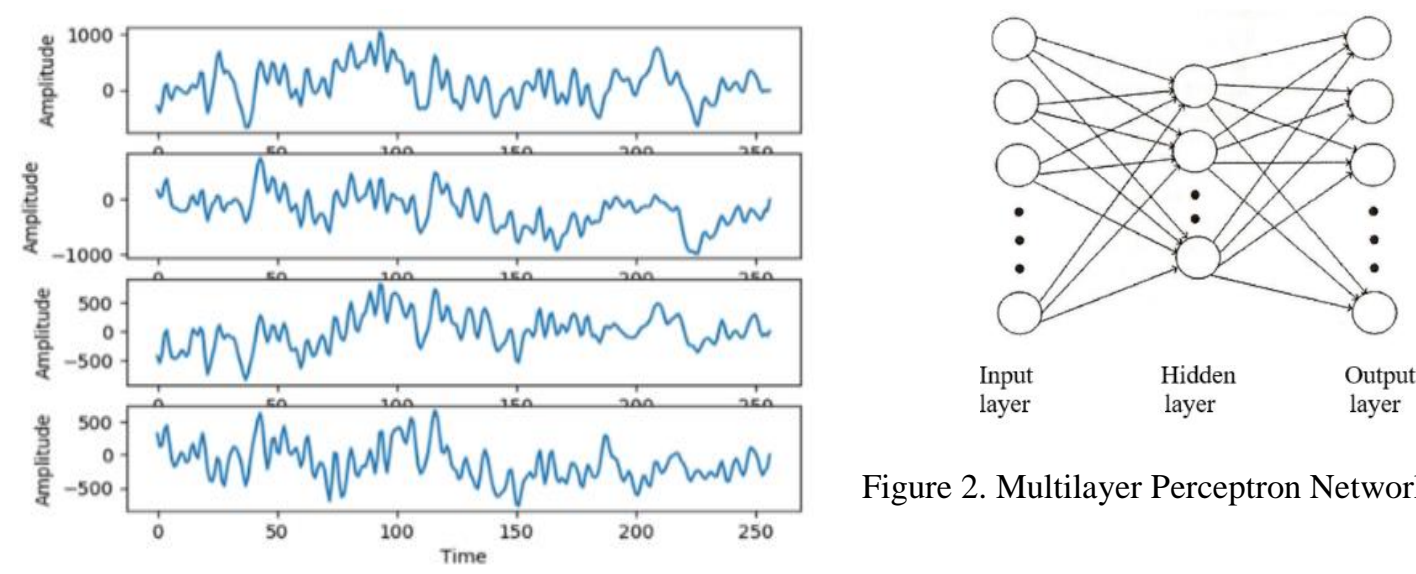

Figure 2. Multilayer Perceptron Network (MLP)

Figure 1. Sample of EEG signal from normal person

There are several layers that are commonly known in CNN such as convolutional layer-which set of learnable filters known as kernel and produce output feature maps that will be output for next layer. Activation layer simplifies back-propagation and reduce redundancies. Pooling layers is to decrease size of feature maps from convolutional layer. Fully connected layer or dense layer is responsible for the output predictions and softmax layer which is an activation function that purpose is to find mean square error. This CNN layers is executed in cascading manner. Figure 3 shows an example of CNN layers from works by [23]. 
There are also feature maps, weights, sample size, Adam optimization and epoch that need to be determined before training process which is common in deep learning. Most time, when more layers are added, the better the learning process which leads to better classification accuracy. For the hidden layers and its output layers, an activation function is needed. Some words such as ReLu, dropout and flatten is relatively new function that exist in CNN application[24-26]. ReLu is the activation function used in every convolution layer, dropout is to prevent overfitting data in fully connected layer and flatten layer that is use to flatten data to $1 \mathrm{D}$ array only.

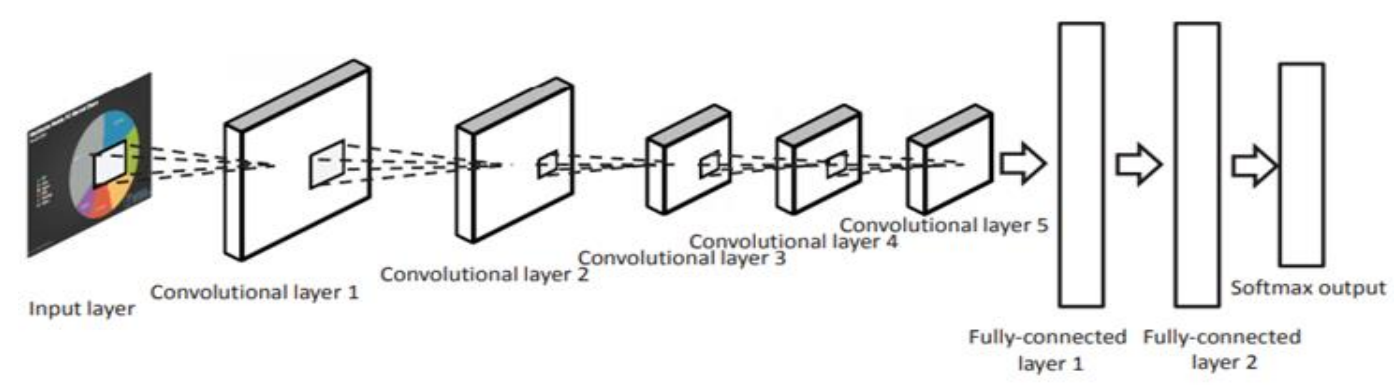

Figure 3. The standard CNN Layer [23]

\section{RESEARCH METHOD}

The methodology for this research is divided into three sections. The first section discusses the existing database, the second section discusses applied pre-processing method and the final section discusses the deep learning design. This system ran on $2.3 \mathrm{GHz}$ Intel Core i5 $2.5 \mathrm{GHz}$ with an NVIDIA GeForce processor that has $610 \mathrm{Mb}$ memory. The Python 3.5.2 language, Open Source Computer Vision Library (OpenCV) and Tensorflow were used for pre-processing and to design the CNN model.

\subsection{Research flowchart}

This research carried out the design of an algorithm to detect autism. It is integrated by using EEG signal. CNN technique will be used in this project as a binary classification. In order to ensure the expected result will be obtained, several major steps need to be conduct such as data collections, implementing, testing, and troubleshooting. These steps are used to analyze the data and output. Figure 4 shows the steps in performing this research.

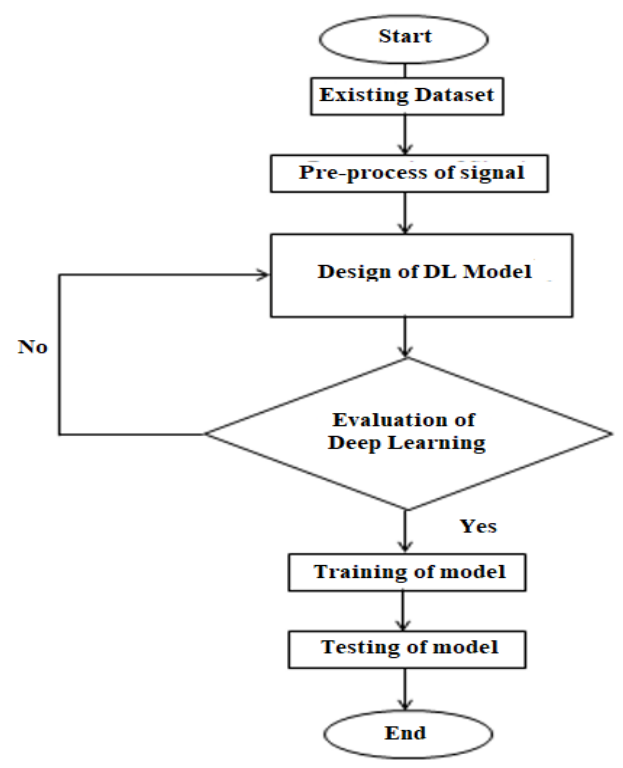

Figure 4. Main development involved 


\subsection{Existing dataset}

The dataset is obtained from existing dataset from other researchers of related paper work. The dataset is request formally by email to researches that have done research by using EEG signal which is obtained from University King Abdul Aziz, Jeddah, Saudi Arabia. The dataset obtained consists of twenty files, 12 normal and 8 for disorders. The dataset was recorded in relaxing state in order to obtain as many artifact-free EEG data as possible. The dataset divided into two groups: normal group (twelves healthy volunteer subjects aged 9-16 years old) and autistic group (eight with autism traits aged 10-16 years old). The EEG signals was acquired at a sampling rate of $256 \mathrm{~Hz}$ using active electrodes and the active digital EEG amplifier and recording system from BCI2000 software. The data acquisition system has 16 channels altogether, which are labeled based on the 10-20 international standard acquisition system.

\subsection{Preprocessing of EEG signal}

Once the dataset is obtained, the raw dataset is pre-processed. The raw dataset obtained is in the form of matrix where it is arranged as such Channel (electrodes number) $\times$ Frequency $(\mathrm{Hz}) \times \mathrm{Time}(\mathrm{s})$. Each of the dataset has an equivalent channel and sampling frequency which is 16 electrodes channel and $256 \mathrm{~Hz}$ frequency respectively. However, the time taken, $t$ of the sampling frequency is different for each dataset. For example, dataset person Normal\#1 is arranged in $16 \times 256 \times 112$, which means the sample is taken for $112 \mathrm{~s}$. For dataset person Normal\#2 is arranged in $16 \times 256 \times 95$ which indicates that the sample is taken for $95 \mathrm{~s}$ only.

All the dataset is pre-processed according to the time taken to record the data. This step is done by using MATLAB software. Thus, for person Normal\#1, it is pre-processed manually for 112 times and is stored in .csv file format. The file is name randomly. For example, at time, $t=1 \mathrm{~s}$ for person Normal\#1, it is label as 'al.csv', where ' $a$ ' is to indicate all file for person Normal\#1 while ' 1 ' indicate the time, $t$ in $1 \mathrm{~s}$. At time, $\mathrm{t}=2 \mathrm{~s}$, the file is save as 'a2.csv'. This is done for all dataset. Table 2 shows the random labeling for each dataset until it completed for the whole dataset consisted of 20 subjects.

Table 2. Random labelling of dataset

\begin{tabular}{cc}
\hline Dataset & Label \\
\hline Normal\#1 & $\mathrm{a}$ \\
Normal\#2 & $\mathrm{b}$ \\
Autism\#1 & $\mathrm{A}$ \\
Autism\#2 & $\mathrm{B}$ \\
\hline
\end{tabular}

Next, the all dataset is combined in a file and is named 'all.csv' file. This makes up a total of 17,136 datasets in a file. All dataset is now at $16 \times 256$ matrix form. The next step of pre-processing is to label the dataset to normal person or autistic patient. For normal person, the label is set to 1 and for autistic patient; it is set to 2 . At the final column, the data is set to either 1 or 2 accordingly based on its classification on either normal or autism. Finally, the data is implemented into a pre-processing algorithm that is used for augmentation and removal of noise using random shuffling and white Gaussian noise.

\subsection{Design of deep learning algorithm}

The deep learning model is designed to fit the EEG data in 2D matrix form. The model is shown in Figure 5 produced in Python. The proposed deep learning model which use CNN architecture has a total of 6 layers which consists of three convolutional layers, one flatten layer and two dense (fully connected) layers. After each convolutional layer, batch normalization is applied. This model also used Gaussian dropout weights. This is because the protocols of transfer learning stated that when CNN is learned from scratch, it needs to be started with random Gaussian distributions. Next, Adam optimization is set to 0.0001 with 200 epochs. Some activation function is also used in this CNN model. The ReLu activation function is used in every convolutional layer while the final dense layer used Softmax activation. The following Table 3 shows how the total number of dataset is distributed for model training, validation and inference.

Table 3. Distribution of dataset

\begin{tabular}{cc}
\hline Model & Distribution (\%) \\
\hline Training Set & $80 \%$ \\
Validation Set & $10 \%$ \\
Test Set & $10 \%$ \\
\hline
\end{tabular}




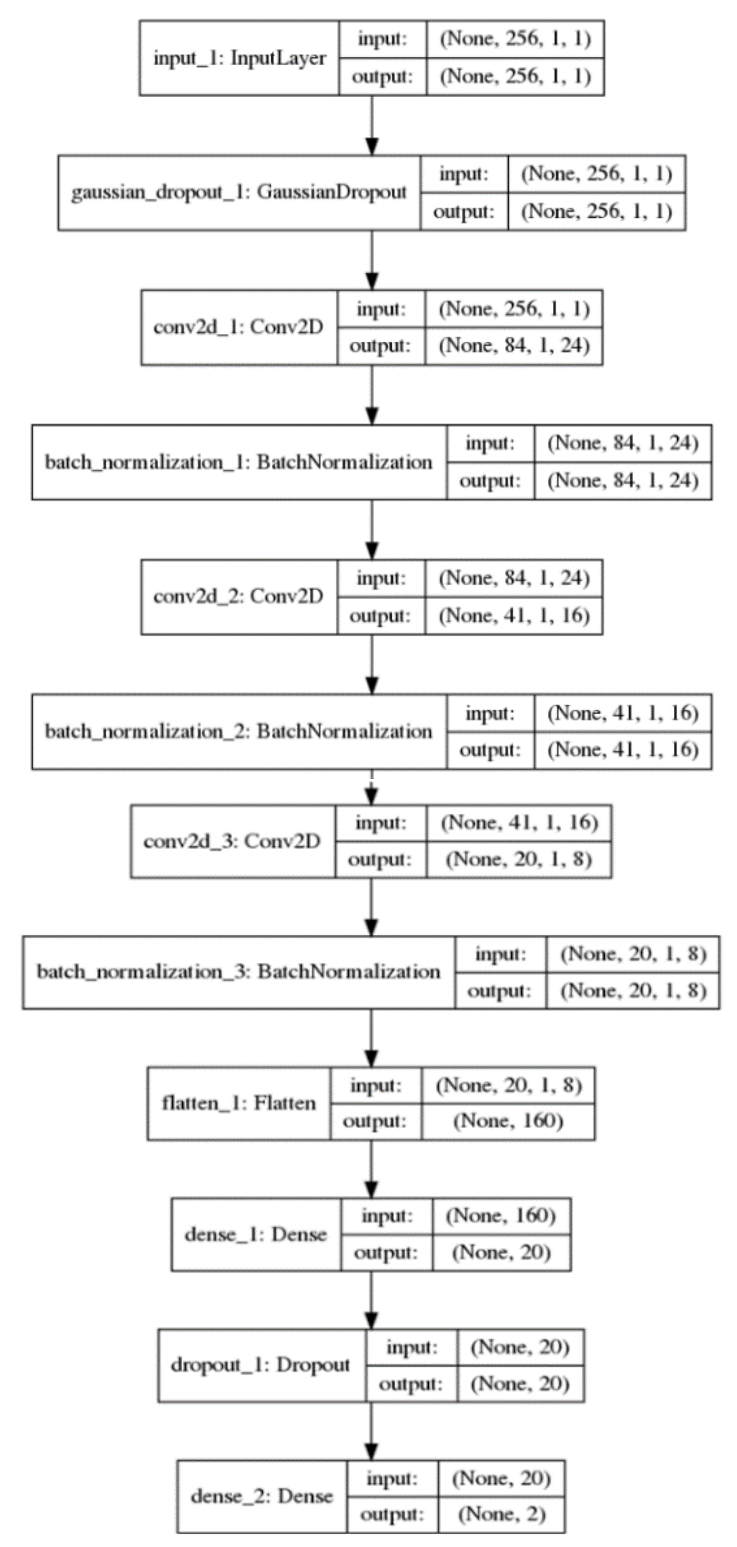

Figure 5. Design of Deep Learning Model

\section{Performance assessment matrix}

Generally, the predictive model derived is retrieved with a number of evaluation measures. For a classification of predicted classes between ASD and Non-ASD, Table 4 shows the possible answer for a test case prediction.

Table 4. Confusion matrix for diagnosis on ASD

\begin{tabular}{ccc}
\hline & \multicolumn{2}{c}{ Predicted Class } \\
\cline { 2 - 3 } Actual Class & ASD & Non-ASD \\
\hline ASD & True Positive (TP) & False Negative (FN) \\
Non-ASD & False Positive (FP) & True Negative (TN) \\
\hline
\end{tabular}

The performance of the classification result is analyzed by the confusion matrix of the test file. The confusion matrix is a particular table layout that allows visualization of the performance of an algorithm. Classification accuracy (1) is one of the common evaluation measures. The number of test cases that have been correctly classified can be identified by using this measure. 


$$
\operatorname{Accuracy}(\%)=\frac{|T P+T N|}{|T P+T N+F P+F N|}
$$

\section{RESULT \& ANALYSIS}

\subsection{Training result}

The training process is repeated for five times. Each time the training process ended, the result of the training is overwriting and automatically stored in a web file. This web file will be updated accordingly when the code training is executed. Thus, when future training is done, the result from previous training is used as a basis for that model training. The first model training achieved accuracy of $70 \%$, the second training achieved accuracy of $79 \%$ and the next three model training achieved a consistent accuracy of $\pm 80 \%$. When a training accuracy did not increase after several training process, this indicate the stopping criterion. The stopping criterion shows that either more dataset need to be added or the CNN model needs to be improved in order to achieve higher accuracy. Figure 6(a) and 6(b) shows training progress of the CNN using the obtained samples, and the respective fluctuations in accuracy and loss metrics across each training epochs.

As shown in the previous figures, the obtained training accuracy is only $80 \%$. Note that the accuracy obtained is based on only twenty datasets and six layers of CNN model. Although convolution to 2D matrix form is possible, the result obtained would not be as well as the result as data trained using in a $2 \mathrm{D}$ image. This is due to the $2 \mathrm{D}$ convolution process is not able to seize completely the spatial relationship between electrodes. This means that EEG data, or basically signal in general, have small amount of spatial regularities for the 2D CNN to derive from.

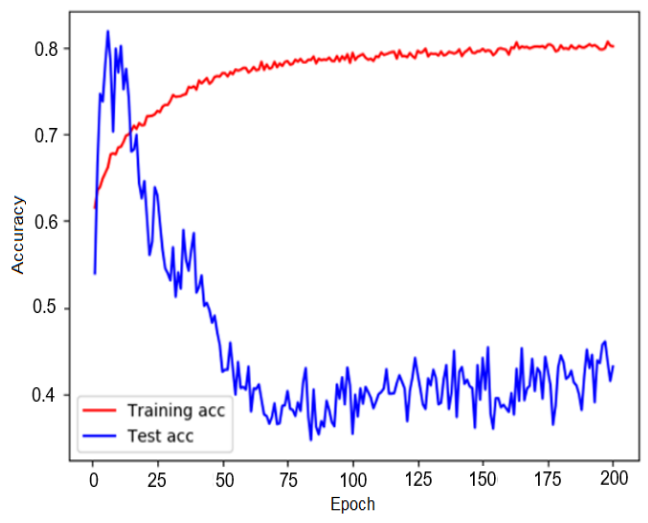

(a)

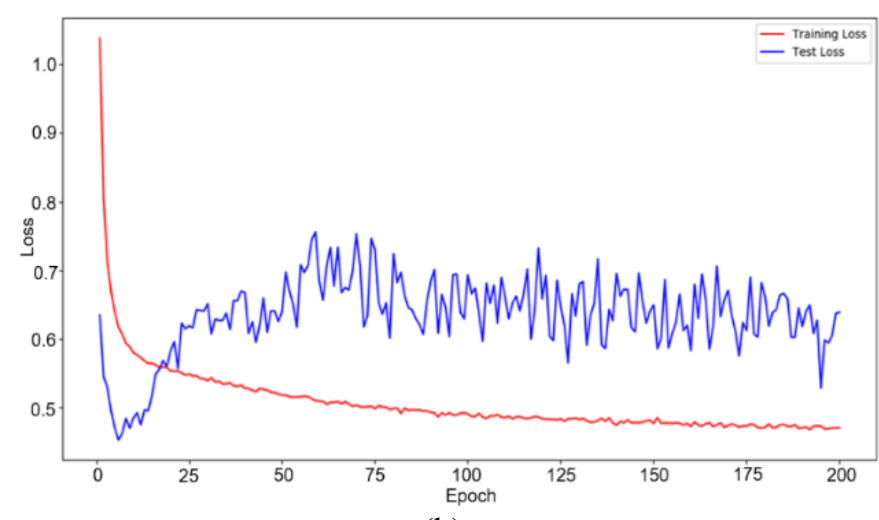

(b)

Figure 6. Training progress, (a) Accuracy, (b) Loss

\subsection{Test result}

An algorithm called the inference algorithm is developed to test whether the developed CNN model can correctly classify a given EEG signal from a random dataset. The inference algorithm loaded the CNN model that is stored in .h5 file. Then an EEG dataset which is either normal or autistic patient EEG is loaded. The predicted output is printed. The design of deep learning model in this project did not achieve high classification of accuracy, thus predicted output produced is rather inconsistent. The produced result sometimes gives a positive result and vice versa. The consistency of the printed result is clearly due to the percentage of accuracy obtained during training set.

\section{CONCLUSIONS}

In conclusion, the objectives of this research are achieved with some improvement that could be made for future studies. Since there is limited dataset available publicly of EEG brain signal on autism patient, the dataset is formally requested from several universities inside and outside the country. The dataset tested in this research obtained from the King Abdulaziz University, Jeddah, Saudi Arabia with total of only 20 persons. The deep learning model using CNN is developed with a total of six layers. As mentioned, each layer in CNN plays major role in ensuring the training and learning process successful. The total of data training is five times with a consistence accuracy rate. The stopping criterion shows that for this specific 
CNN model, the maximum achievable accuracy is around eighty percent only. The evaluation of the deep learning model shows inconsistency of the printed result which is the direct cause of the low accuracy. Although the achieved accuracy is only about $80 \%$, this shows that it has higher potential in developing better algorithm by using more complex deep learning model and having more dataset.

The major contribution of this research towards the society is in producing an alternative method to make detection on the presence of autism in a child. The current diagnose method of ASD is very much time consuming, thus it is not reliable since studies have shown that ASD child suffers from many side effect of ASD since young age such as visual impairment. Furthermore, a fully develop and high accuracy system could be employed by the Ministry of Health as a new diagnosing method of ASD to high risk children based on EEG. In terms of economic, it can greatly reduce the time taken to detect ASD thus early treatment could be planned by pediatrics to provide better health support to the autistic patient.

\section{ACKNOWLEDGEMENTS}

Authors are grateful to Universiti Teknikal Malaysia Melaka for the financial support through fundamental research grant FRGS/2018/FKEKK-CERIA/F00363. Authors also want to thank researcher at King Abdulaziz University, Jeddah, Saudi Arabia in providing the dataset.

\section{REFERENCES}

[1] E. B. Johnson, Autism Spectrum Disorders: The Worldwide Charm And Challenge Of Autism Spectrum Disorders, Special Ed. Bradford, Uk: Emerald Group Publishing, 2014.

[2] M. Sharda Et Al., "Language Ability Predicts Cortical Structure And Covariance In Boys With Autism Spectrum Disorder," Cereb. Cortex, Vol. 27, No. 3, Pp. 1849-1862, 2017.

[3] J. Jennings Dunlap, “Autism Spectrum Disorder Screening And Early Action,” J. Nurse Pract., Vol. 15, No. 7, Pp. 496-501, 2019.

[4] T. Liu, Y. Chen, D. Chen, C. Li, Y. Qiu, And J. Wang, "Altered Electroencephalogram Complexity In Autistic Children Shown By The Multiscale Entropy Approach,” Neuroreport, Vol. 28, No. 3, Pp. 169-173, 2017.

[5] P. R. P. Hoole Et Al., "Autism, Eeg And Brain Electromagnetics Research," 2012 Ieee-Embs Conf. Biomed. Eng. Sci. Iecbes 2012, No. December, Pp. 541-543, 2012.

[6] N. Fauzan And N. H. Amran, "Brain Waves And Connectivity Of Autism Spectrum Disorders," Procedia - Soc. Behav. Sci., Vol. 171, Pp. 882-890, 2015.

[7] L. Bote-Curiel, S. Muñoz-Romero, A. Gerrero-Curieses, And J. L. Rojo-Álvarez, "Deep Learning And Big Data In Healthcare: A Double Review For Critical Beginners," Appl. Sci., Vol. 9, No. 11, 2019.

[8] F. C. Morabito Et Al., "Deep Convolutional Neural Networks For Classification Of Mild Cognitive Impaired And Alzheimer's Disease Patients From Scalp Eeg Recordings," 2016 Ieee 2nd Int. Forum Res. Technol. Soc. Ind. Leveraging A Better Tomorrow, Rtsi 2016, 2016.

[9] W. H. L. Pinaya, A. Mechelli, And J. R. Sato, "Using Deep Autoencoders To Identify Abnormal Brain Structural Patterns In Neuropsychiatric Disorders: A Large-Scale Multi-Sample Study,” Hum. Brain Mapp., Vol. 40, No. 3 , Pp. 944-954, 2019.

[10] G. Cho, J. Yim, Y. Choi, J. Ko, And S. H. Lee, "Review Of Machine Learning Algorithms For Diagnosing Mental Illness,” Psychiatry Investig., Vol. 16, No. 4, Pp. 262-269, 2019.

[11] W. Bosl, A. Tierney, H. Tager-Flusberg, And C. Nelson, "Eeg Complexity As A Biomarker For Autism Spectrum Disorder Risk," Bmc Med., Vol. 9, 2011.

[12] M. S. Mythili And A. R. M. Shanavas, "A Study On Autism Spectrum Disorders Using Classification Techniques," Ijcsit, Vol. 5, No. 6, Pp. 7288-7291, 2014.

[13] J. B. Ewen Et Al., "Decreased Modulation Of Eeg Oscillations In High-Functioning Autism During A Motor Control Task," Vol. 10, No. May, Pp. 1-11, 2016.

[14] T. Heunis Et Al., "Recurrence Quantification Analysis Of Resting State Eeg Signals In Autism Spectrum Disorder A Systematic Methodological Exploration Of Technical And Demographic Confounders In The Search For Biomarkers," Bmc Med., Vol. 16, No. 1, Pp. 1-17, 2018.

[15] I. Vasilev, D. Slater, G. Spacagna, P. Roelants, And V. Zocca, Python Deep Learning: Exploring Deep Learning Techniques And Neural Network Architectures With Pytorch, Keras And Tensorflow, 2nd Editio. Birmingham, Uk: Packt Publishing, 2018.

[16] M. J. Alhaddad Et Al., "Diagnosis Autism By Fisher Linear Discriminant Analysis Flda Via Eeg," Vol. 4, No. 2 , Pp. 45-54, 2012.

[17] Gavin Hackeling, Mastering Machine Learning With Scikit-Learn Second, 2nd Editio. Packt Publishing, 2017.

[18] P. Marzuki, A. R. Syafeeza, A. N. Alisa, And M. K. M. F. Alif, "An Improved Of Malaysian License Plates Detection Using Deep Convolutional Neural Networks," Symp. Electr. Mechatronics Appl. Sci. 2018, Vol. 2018, No. November, Pp. 75-76, 2018.

[19] N. A. Ali, A. R. Syafeeza, L. J. Geok, Y. C. Wong, N. A. Hamid, And A. S. Jaafar, "Design Of Automated Computer-Aided Classification Of Brain Tumor Using Deep Learning," In Intelligent And Interactive Computing, 2019, Pp. 285-291. 
[20] A. R. Syafeeza, S. S. Liew, And R. Bakhteri, "Convolutional Neural Network For Face Recognition With Pose And Illumination Variation," Int. J. Eng. Technol. Convolutional, Vol. 6, No. 1, Pp. 44-57, 2014.

[21] M. K. M. F. Alif, P. M. , A.R.Syafeeza, And N. A. Ali, "Fused Convolutional Neural Network For Facial Expression Recognition,” Proc. Symp. Electr. Mechatronics Appl. Sci. 2018, Pp. 73-74, 2018.

[22] R. Djemal, K. Alsharabi, S. Ibrahim, And A. Alsuwailem, "Eeg-Based Computer Aided Diagnosis Of Autism Spectrum Disorder Using Wavelet, Entropy, And Ann,” Biomed Res. Int., Vol. 2017, 2017.

[23] B. Tang Et Al., "Deepchart: Combining Deep Convolutional Networks And Deep Belief Networks In Chart Classification,” Signal Processing, Vol. 124, Pp. 156-161, 2016.

[24] S. S. B, D. Mahapatra, Z. Ge, And R. Chakravorty, Learning For Weakly Supervised Localization Of Chest Pathologies In X-Ray Images, Vol. 1. Springer International Publishing, 2018.

[25] M. A. Bujang And T. H. Adnan, "Requirements For Minimum Sample Size For Sensitivity And Specificity Analysis,” J. Clin. Diagnostic Res., Vol. 10, No. 10, Pp. Ye01-Ye06, 2016.

[26] H. Rajaguru, "Analysis Of Pac Learning Based Bayesian Optimization With Autoencoders For Epilepsy Classification From Eeg Signals," vol. 8, no. 12, pp. 206-212, 2017.

\section{BIOGRAPHIES OF AUTHORS}
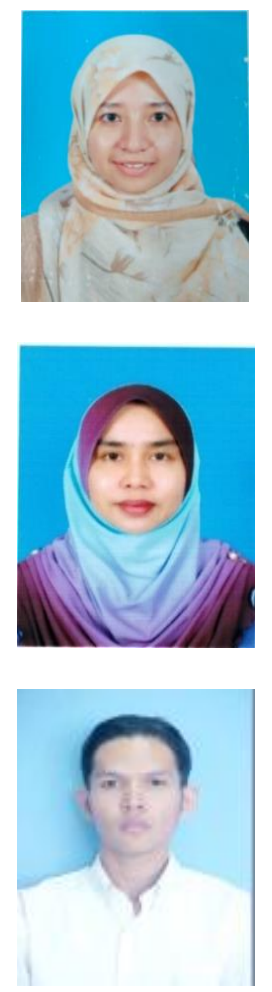

Nur Alisa Ali currently is a PhD student in Universiti Teknikal Malaysia Melaka (UTeM), Malaysia. She received her Higher National Diploma (HND) in Electronics Engineering from British Malaysian Institute, UniKL-BMI (2003) and BEng in Electronics from University of Surrey(UNiS), United Kingdom in 2006. She received her Master Degree in Computer Systems from University of South Australia (UniSA), Australia in 2009. Currently, her PhD research involves embedded systems, machine and deep learning; image and signal processing.

Dr. Syafeeza Ahmad Radzi received her B. Eng degree in Electrical-Electronic Engineering in 2003 and her M. Eng degree in Electrical - Electronic \& Telecommunication Engineering in 2005 from Universiti Teknologi Malaysia. She also received her Ph.D degree in Electrical Engineering from the same university in 2014. She is currently a Senior Lecturer at the Faculty of Electronic Engineering and Computer Engineering, Universiti Teknikal Malaysia Melaka (UTeM). She has been an academician in UTeM since 2006. She dedicates herself to university teaching and conducting research. Her research fields include embedded system, pattern recognition, machine learning, deep learning, image processing, biometric, etc.

Dr. Abd Shukur Ja'afar received both first and master degree from Universiti Teknologi Malaysia (UTM) in Bachelor of Electrical Engineering (2002) and Master of Engineering in Electronic and Telecommunication (2005). He joined Universiti Teknikal Malaysia Melaka (UTeM) as a lecturer in 2005 and received PhD in Communication System from Lancaster University, UK. Currently his research interest on RF, Microwave circuits and algorithm development for indoor positioning and navigation. 